\title{
Fornix-based versus limbal-based conjunctival flaps in trabeculectomy with mitomycin C in high-risk patients
}

This article was published in the following Dove Press journal:

Clinical Ophthalmology

15 May 2014

Number of times this article has been viewed

\author{
Utako Kuroda \\ Toshihiro Inoue \\ Nanako Awai-Kasaoka \\ Kohei Shobayashi \\ Sachi Kojima \\ Hidenobu Tanihara \\ Department of Ophthalmology, \\ Faculty of Life Sciences, Kumamoto \\ University, Kumamoto, Japan
}

Purpose: To compare the surgical outcomes of limbal-based and fornix-based trabeculectomy in eyes with a history of ocular incisional surgery.

Methods: Twenty-six eyes underwent limbal-based trabeculectomy (group LB), and were condition matched with 26 eyes that received fornix-based trabeculectomy (group FB). Surgical failure was recorded retrospectively if the intraocular pressure value was either $\geq 21, \geq 18$, and $\geq 15 \mathrm{mmHg}$ (conditions $\mathrm{A}, \mathrm{B}$, and $\mathrm{C}$, respectively) or $<4 \mathrm{mmHg}$ or if the patient required additional glaucoma surgery. Kaplan-Meier survival curve analysis was used to assess surgical failure.

Results: For condition A, the 2 year surgical success probabilities were $75.0 \%$ and $63.9 \%$ in groups FB and LB, respectively ( $P=0.124)$. The corresponding values were $55.0 \%$ and $61.7 \%$ $(P=0.638)$ in condition $\mathrm{B}$, and $55.0 \%$ and $57.0 \%(P=0.454)$ in condition $\mathrm{C}$. The rates of bleb leakage, hypotony, choroidal detachment, and bleb-related infection were $11.5 \%, 26.9 \%, 50.0 \%$, and $7.7 \%$ in group LB, respectively. The corresponding values in group FB were $30.8 \%, 23.1 \%$, $46.2 \%$, and $0.0 \%$, which were not statistically different between the two groups.

Conclusion: No significant differences in surgical outcomes were observed between limbal-based and fornix-based trabeculectomy for patients with a history of incisional ocular surgeries.

Keywords: trabeculectomy, previous surgery, surgical outcomes, surgical complications

\section{Introduction}

Trabeculectomy is one of the major therapeutic modalities in the management of glaucoma, ${ }^{1}$ because of its highly reliable effects on lowering intraocular pressure (IOP). Although the techniques have been established for nearly 50 years, the use of various options still remains under debate, including whether to use mitomycin $\mathrm{C}$ (MMC), or 5 fluorouracil, single or combined surgery, thin or thick scleral flaps, or limbal- or fornix-based conjunctival flaps. Of these different options, the manner of the conjunctival incision is known to affect bleb morphology. Past studies have reported that eyes that received limbal-based trabeculectomy tended to develop cystic blebs when compared with those that underwent the fornix-based technique, ${ }^{2-4}$ and that these differences in bleb morphology may cause different surgical outcomes. With regard to this issue, a number of studies have compared the post-operative outcomes, including IOP control and surgical complications, between these two types of conjunctival incisions. ${ }^{4-7}$ However, no consensus has been reached on the question as to which technique is better for high-risk cases.

We previously reported various risk factors for the surgical success of trabeculectomy with MMC. ${ }^{8-12}$ In these previous studies, background factors that could
Correspondence:Toshihiro Inoue Department of Ophthalmology, Faculty of Life Sciences, Kumamoto University, I-I-I Honjo, Kumamoto, Japan Tel +8I 963735247

Fax +8I 963735249

Email noel@da2.so-net.ne.jp 
potentially enhance subconjunctival scarring (eg, a history of phacoemulsification in eyes with either primary open-angle glaucoma or uveitic glaucoma; a history of vitrectomy in neovascular glaucoma eyes; or a history of granulomatous uveitis in eyes with uveitic glaucoma) were repeatedly identified as prognostic factors, suggesting that exaggerated wound healing around the scleral flap causes surgical failure in the control of IOP after trabeculectomy. Thus, eyes with such risk factors are considered high-risk cases for trabeculectomy and therefore to require optimized surgical techniques to lower the IOP. However, to our knowledge, no study has directly compared the post-operative results of limbal-based trabeculectomy with fornix-based trabeculectomy in highrisk and condition-matched populations. In this study, we compared the post-operative outcomes between these two types of conjunctival incisions during trabeculectomy in eyes with a history of cataract and/or glaucoma surgery.

\section{Materials and methods Patient population}

The study was conducted in accordance with the Declaration of Helsinki, and was approved by the Institutional Review Board of Kumamoto University. Medical records were retrospectively reviewed, and all patients who received limbalbased trabeculectomy at Kumamoto University Hospital between April 2004 and December 2010 were identified. Of these consecutive patients, those who had eyes with histories of cataract and/or glaucoma surgeries were included, and were categorized as "group LB". Exclusion criteria were eyes with histories of vitrectomy and/or with ocular ischemic diseases, including proliferative diabetic retinopathy. When both eyes in a patient met the criteria, we included only the eye that had received objective trabeculectomy. We then matched the eyes that had undergone fornix-based trabeculectomy (defined as "group FB") to those in group LB according to age, sex, etiology of glaucoma, intraocular surgical history, and the preoperative IOP.

\section{Surgical technique}

Trabeculectomy was performed as previously reported..$^{8,9}$ Briefly, a limbal- or fornix-based conjunctival flap was made, followed by a triangular single half-layer scleral flap. Small sponges soaked in MMC $(0.4 \mathrm{mg} / \mathrm{mL})$ were applied to the exposed tissues for 4 minutes. After the removal of the sponges, the wound was irrigated with $200 \mathrm{~mL}$ of balanced salt solution. A small trabecular block was excised, and a fistula leading to the anterior chamber was created. A peripheral iridectomy was then performed. The scleral flap and the conjunctiva were closed with a 10-0 nylon suture. Postoperatively, all patients received a similar topical medical regimen: $1 \%$ topical atropine sulfate for 1 week, $0.1 \%$ topical betamethasone and levofloxacin for 6-12 weeks. Laser suture lysis was conducted on the basis of the IOP value and the bleb condition.

\section{Main outcome measure and observation procedure}

For avoiding the effect of short-term postoperative IOP fluctuations in the analysis, IOP values 2 months after trabeculectomy or later were adopted. We defined surgical failure as an IOP value of $\geq 21, \geq 18$, and $\geq 15 \mathrm{mmHg}$ (conditions A, B, and C, respectively), with or without the use of topical ocular hypotensive medications, and verified the values at the next visit. For all conditions, an IOP value of $<4 \mathrm{mmHg}$ and additional glaucoma surgery (except needling) were also defined as surgical failure. IOP values were measured with a Goldmann applanation tonometer. The preoperative IOP value for each eye was determined as the average of three measurements before trabeculectomy.

\section{Statistical analysis}

Data were analyzed using the JMP version 8 statistical package program (Cary, NC). An unpaired 2-tailed -test and the chi-square test were used to compare characteristics between the two groups. We used Kaplan-Meier survival curve analysis to assess surgical failure, with $P$-values derived from the log-rank test. A $P$-value of $<0.05$ was considered statistically significant.

\section{Results}

\section{Preoperative patient characteristics}

In each group, 26 eyes of 26 patients satisfied the inclusion criteria for the study. The characteristics of the two groups are shown in Table 1. No significant differences were observed between the two groups in terms of age, sex, etiology of glaucoma, intraocular surgical history, preoperative IOP values, and the observational period.

\section{Cumulative probability of surgical success}

Figure 1A presents the results of the Kaplan-Meier survival analysis for both groups under condition A (success, IOP $<21 \mathrm{mmHg}$ ). The probability of surgical success at 1 year after trabeculectomy was $86.7 \%$ and $63.9 \%$ in groups FB and LB, respectively. Two years after trabeculectomy, the corresponding values were $75.0 \%$ and $63.9 \%$. The statistical analysis showed no significant difference between the two groups $(P=0.124)$. When the cut-off IOP value was 
Table I Baseline characteristics of all patients who underwent trabeculectomy with mitomycin C

\begin{tabular}{|c|c|c|c|}
\hline Characteristic & Group LB & Group FB & $P$-value \\
\hline Number of patients & 26 & 26 & - \\
\hline Male/female & $|5 /| \mid$ & $15 / 11$ & 1.00 \\
\hline \multicolumn{4}{|l|}{ Age (years) } \\
\hline Mean \pm SD & $59.8 \pm 21.4$ & $62.0 \pm 21.5$ & 0.81 \\
\hline Range & 4 to 85 & 0 to 86 & \\
\hline \multicolumn{4}{|l|}{ Preoperative IOP (mmHg) } \\
\hline Mean \pm SD & $29.4 \pm 6.9$ & $30.6 \pm 7.2$ & 0.68 \\
\hline Range & 13.7 to 42.0 & 18.0 to 45.7 & \\
\hline \multicolumn{4}{|c|}{ Number of preoperative glaucoma drops } \\
\hline Mean $\pm S D$ & $2.7 \pm 0.6$ & $2.8 \pm 0.7$ & 0.54 \\
\hline Range & I to 4 & I to 4 & \\
\hline \multicolumn{4}{|l|}{ Follow-up period (months) } \\
\hline Mean \pm SD & $29.8 \pm 27.1$ & $38.3 \pm 24.0$ & 0.22 \\
\hline Range & 1.0 to 96.4 & 0.4 to 87.2 & \\
\hline \multicolumn{4}{|l|}{ Etiology of glaucoma } \\
\hline Exfoliation glaucoma & $12(46 \%)$ & II (42\%) & 0.78 \\
\hline Primary open-angle glaucoma & $5(19 \%)$ & $7(27 \%)$ & 0.51 \\
\hline Uveitic glaucoma & $5(19 \%)$ & $5(19 \%)$ & 1.00 \\
\hline Developmental glaucoma & $4(15 \%)$ & $3(12 \%)$ & 0.68 \\
\hline \multicolumn{4}{|l|}{ History of previous ocular surgery } \\
\hline Trabeculectomy & $12(46 \%)$ & $12(46 \%)$ & 1.00 \\
\hline Trabeculotomy & $9(28 \%)$ & $8(3 \mid \%)$ & 0.77 \\
\hline Cataract surgery & $17(65 \%)$ & $14(54 \%)$ & 0.41 \\
\hline
\end{tabular}

Abbreviations: $F B$, fornix-based; IOP, intraocular pressure; LB, limbal-based; SD, standard deviation.

defined as $\geq 18 \mathrm{mmHg}$ (condition $\mathrm{B}$ ), the probability of 1 year success was $66.2 \%$ in group FB and $61.7 \%$ in group LB, while the probability of 2 year success was $55.0 \%$ in group FB and $61.7 \%$ in group LB (Figure 1B). No significant difference in the probability of success was found between the two groups $(P=0.638)$. For condition $\mathrm{C}(\mathrm{IOP} \geq 15 \mathrm{mmHg})$, the probability of 1 year success was $66.2 \%$ and $57.0 \%$ in groups FB and LB, respectively, and the corresponding probability of 2-year success was $55.0 \%$ and $57.0 \%$ (Figure 1C). No significant difference was found between the groups $(P=0.454)$. Under condition $\mathrm{A}$, three eyes in group FB and seven eyes in group LB were defined as surgical failure because of excessive IOP values. The corresponding number of eyes were nine and nine under condition $\mathrm{B}$ and nine and ten under condition $\mathrm{C}$. One eye in each group fulfilled the criteria of surgical failure because of additional glaucoma surgery under condition A. For both conditions B and C, one eye in group LB was defined as failure for the same reason. For all three conditions, one eye in group LB met the criteria for surgical failure because of hypotony.

\section{Postoperative IOP value and the number of glaucoma eye drops}

One year after trabeculectomy, the mean IOP values were $12.8 \pm 6.5 \mathrm{mmHg}$ in group $\mathrm{FB}$ (20 eyes) and 11.6 $\pm 5.9 \mathrm{mmHg}$ in group LB (19 eyes); the number of IOP-reducing eye drops was $0.3 \pm 0.7$ in group FB and $0.8 \pm 1.3$ in group LB (Table 2). Two years after trabeculectomy, the mean IOP values were $13.7 \pm 7.3 \mathrm{mmHg}$ in group $\mathrm{FB}$ (16 eyes) and $12.2 \pm 5.4 \mathrm{mmHg}$ in group LB (13 eyes), and the number of IOP-reducing eye drops was $0.3 \pm 0.8$ in group $\mathrm{FB}$ and $0.6 \pm 1.3$ in group LB (Table 2). No significant differences were observed in both parameters at either 1 or 2 years after trabeculectomy between the two groups.

\section{Surgical complications}

No severe complications occurred during surgery. Table 3 summarizes the postoperative complications during the follow-up period. Leaks of aqueous fluid were seen in 3 eyes (11.5\%) in group LB and 8 eyes $(30.8 \%)$ in group FB $(P=0.090)$. Meanwhile, choroidal detachments were found in 7 eyes $(26.9 \%)$ and 6 eyes $(23.1 \%)$ in groups LB and FB, respectively $(P=0.749)$. Hypotony (IOP $\leq 4)$ occurred in 13 eyes $(50.0 \%)$ in group LB and 12 eyes $(46.2 \%)$ in group FB $(P=0.781)$ during the observational period. Two eyes $(7.7 \%)$ in group LB suffered mild bleb-related infection, which was relieved with only topical antibiotics. No eye in group FB experienced bleb-related infections. No statistical difference was found between the two groups in the incidence ratio of each postoperative complication. 
A

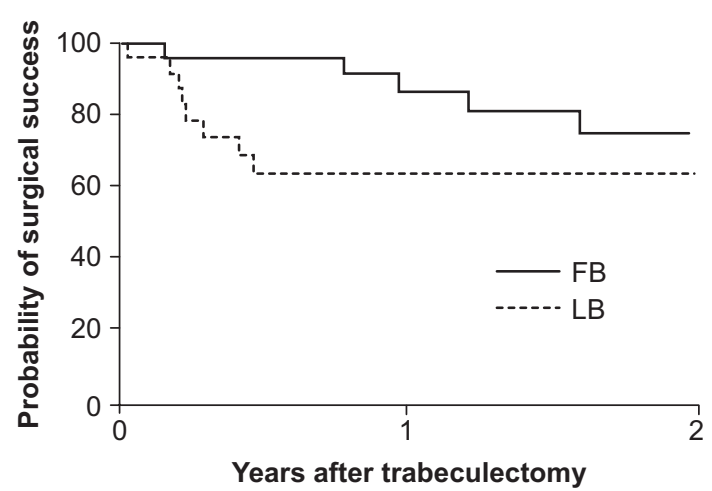

B

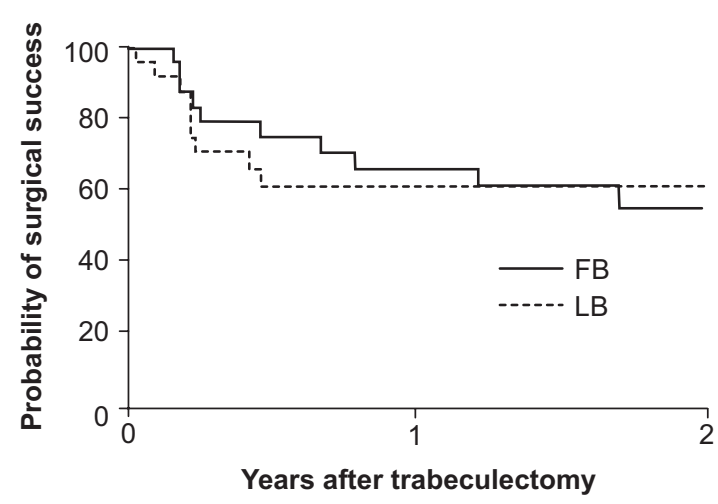

C

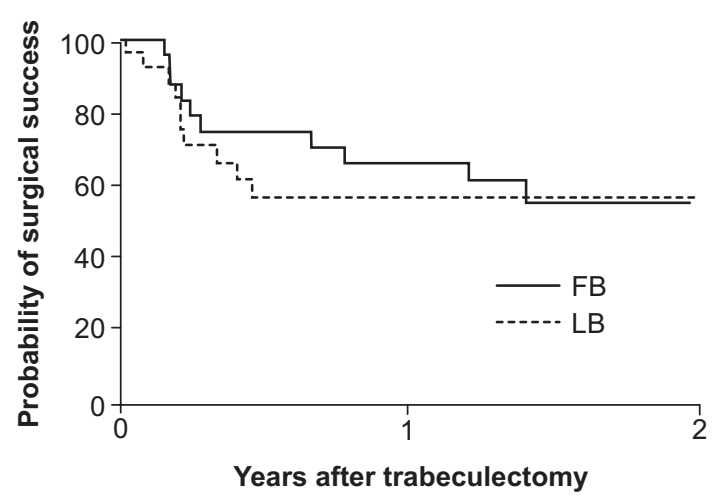

Figure I Kaplan-Meier survival plots of the study groups.

Notes: Kaplan-Meier survival plots of the study groups according to the manner of the conjunctival flap: fornix-based (FB, solid line) or limbal-based (LB, dotted line). Both groups consisted of 26 eyes of 26 patients. (A) Surgical failure was defined as an intraocular pressure (IOP) value of $\geq 21$, an IOP value of $<4 \mathrm{mmHg}$, and additional glaucoma surgery. The probability of success of the trabeculectomy was not significantly different between groups $(P=0.124)$. (B) Surgical failure was defined as an IOP value of $\geq 18$, an IOP value of $<4 \mathrm{mmHg}$, and additional glaucoma surgery. The probability of success of the trabeculectomy was not significantly different between groups $(P=0.638)$. (C) Surgical failure was defined as an IOP value of $\geq 15$, an IOP value of $<4 \mathrm{mmHg}$, and additional glaucoma surgery. The probability of success of the trabeculectomy was not significantly different between groups $(P=0.454)$.

\section{Discussion}

The present study revealed an absence of significant differences in the surgical outcomes, the probabilities of success, and the rates of surgical complications between limbal-based and fornix-based trabeculectomy in patients with conjunctival
Table 2 Comparison of postoperative clinical outcomes between groups LB and FB

\begin{tabular}{llll}
\hline & Group LB & Group FB & $P$-value* \\
\hline $\begin{array}{l}\text { One year after trabeculectomy } \\
\text { Number of patients }\end{array}$ & 19 & 20 & \\
IOP (mean \pm SD; mmHg) & $11.6 \pm 5.9$ & $12.8 \pm 6.5$ & 0.55 \\
$\quad \begin{array}{l}\text { Number of glaucoma eyedrops } \\
\text { (mean } \pm \text { SD) }\end{array}$ & $0.8 \pm 1.3$ & $0.3 \pm 0.7$ & 0.12 \\
Two years after trabeculectomy & & & \\
$\quad$ Number of patients & 13 & 16 & \\
IOP (mean \pm SD; $m$ mHg) & $12.2 \pm 5.4$ & $13.7 \pm 7.3$ & 0.53 \\
$\quad \begin{array}{l}\text { Number of glaucoma eyedrops } \\
\text { (mean } \pm \text { SD) }\end{array}$ & $0.6 \pm 1.3$ & $0.3 \pm 0.8$ & 0.45 \\
\hline
\end{tabular}

Notes: *A two-tailed $t$-test and the Wilcoxon test were used to evaluate differences between groups in terms of the IOP values and the number of glaucoma drops, respectively.

Abbreviations: FB, fornix-based; IOP, intraocular pressure; LB, limbal-based; $\mathrm{SD}$, standard deviation.

scars due to cataract and/or glaucoma surgeries. To our knowledge, this report is the first one to compare the surgical outcomes of limbal-based and fornix-based trabeculectomy in eyes with conjunctival scars. Although the present study was a retrospective one and included a limited number of eyes, these data provide insight into decision making on a particular technical option (eg, the type of conjunctival flap) in trabeculectomy, since the characteristics (eg, age, sex, etiology of glaucoma) of the two groups were highly similar. In addition, the patients in the group LB were consecutive cases, and were therefore nearly free from selection bias.

Our previous studies indicated that a history of past trabeculectomy or phacoemulsification increased the probability of surgical failure in subsequent trabeculectomy. ${ }^{9,13,14}$ The present study can be considered to reveal the surgical results of trabeculectomy in high-risk eyes. We found no statistical difference between groups FB and LB in regard to the probability of success, the IOP value, and the number of glaucoma eyedrops after trabeculectomy, suggesting the IOP-lowering effect was similar between the groups. Although various comparative studies have found no differences in the IOP-lowering effect between limbal-based and fornix-based trabeculectomy with or

Table 3 Comparison of the incidence ratio of the postoperative complications between groups LB and FB

\begin{tabular}{llll}
\hline Complication & Group LB & Group FB & P-value* \\
\hline Bleb leak & $3(12 \%)$ & $8(31 \%)$ & 0.09 \\
Hypotony (IOP $\leq 4)$ & I $3(50 \%)$ & $12(46 \%)$ & 0.78 \\
Choroidal detachment & $7(27 \%)$ & $6(23 \%)$ & 0.75 \\
Bleb-related infection & $2(8 \%)$ & 0 & 0.15 \\
\hline
\end{tabular}

Note: *chi-square test.

Abbreviations: $\mathrm{FB}$, fornix-based; IOP, intraocular pressure; LB, limbal-based. 
without an antimetabolite, ${ }^{4-7}$ data on the surgical results of trabeculectomy in high-risk eyes have been limited. Wells et al analyzed the surgical results of glaucoma patients aged less than 30 years, and found the IOP value after fornix-based trabeculectomy tended to be higher than that after limbal-based trabeculectomy; however, the difference was not statistically significant. ${ }^{4}$ This previous study was considered as postsurgical data for high-risk eyes, since the younger age was thought to be a risk factor for the failure of filtration surgery. ${ }^{15-17}$ Collectively, the results of this previous study and the current one indicate the IOP-lowering effect of limbal-based trabeculectomy is similar to that of fornix-based trabeculectomy even in high-risk eyes. Since the sample number was limited and the study design was retrospective in the present study, randomized prospective studies with larger sample required in the future.

Bleb leaks are sometimes observed at the early postsurgical stage, and are thought to increase the risk of blebrelated infection, especially at the late post-surgical stage. Several studies have reported that early bleb leaks (within 1-6 weeks after trabeculectomy) occurred more frequently in fornix-based trabeculectomy compared with limbal-based trabeculectomy. ${ }^{3,6,7}$ Meanwhile, another report found no difference between these two groups in the occurrence ratio of bleb leaks. ${ }^{1}$ In the present study, although the rate in group FB $(31 \%)$ tended to be high compared with that of group LB $(12 \%)$, the difference was not significant, possibly because of the small sample number. When these various results are considered collectively, the limbal-based conjunctival flap might be favorable for a secure wound closure. However, further studies with a large sample size are required to conclude whether the occurrence rate is dependent on the manner of conjunctival flap in high-risk eyes.

A previous study showed a higher rate of postsurgical hypotony in fornix-based trabeculectomy than in limbal-based trabeculectomy. ${ }^{7}$ On the other hand, several studies reported no difference between the 2 techniques in the incidence ratio of hypotony. ${ }^{4-6}$ In the present study, the occurrence ratio of hypotony was similar between the groups: $50 \%$ and $46 \%$ in groups LB and FB, respectively. The corresponding values for choroidal detachment were $27 \%$ and $23 \%$. Since choroidal detachment usually occurred in conjunction with hypotony, the occurrence rates of the choroidal detachment may reflect those of hypotony. Further, the results indicated that the ratio of choroidal detachment to hypotony was independent of the manner of the conjunctival flap. In the present study, the target IOPs at the early postsurgical stage were relatively low. Therefore, the occurrence ratios of these complications may become high in eyes that have undergone trabeculectomy for both types of conjunctival flap.

Bleb-related infection is a rare but serious complication after trabeculectomy. Since the occurrence rate was not high, a large-scale study is required for meaningful comparisons among groups. In one large trial analyzing 439 trabeculectomy sites in 347 patients, bleb-related infections were reported to occur more frequently in limbal-based than in fornix-based trabeculectomy during a period of 4 years after trabeculectomy, although the difference was statistically marginal $(P=0.054){ }^{7}$ Another large study that included 908 eyes of 908 Japanese patients revealed that the cumulative probability was $1.5 \%$ in both types of conjunctival flaps at a 2.5 -year follow up. ${ }^{18}$ In another study, no bleb-related infection occurred in 82 cases, which included fornix- and limbal-based trabeculectomies, during a follow-up period of 20 months after surgery. ${ }^{6}$ The present study had two cases $(8 \%)$ of bleb-related infection in group LB during a period of 2 years after trabeculectomy. In contrast, no bleb-related complications were observed in group FB. Regarding the occurrence rate of bleb-related infection, no firm conclusion can be drawn from the present study because of the small sample size, as described above. Furthermore, the relatively high rate of total cases (4\%, two cases in 52 eyes) might have been due to preexisting conjunctival scars caused by previous incisional ocular surgeries.

\section{Conclusion}

No significant differences in terms of surgical success and complication rates were found during a 2-year follow-up between limbal-based and fornix-based trabeculectomy for patients with a history of incisional ocular surgeries.

\section{Acknowledgments}

This work was supported in part by JSPS KAKENHI Grant Numbers 23390403, 23659814, and 23791994; the sponsor or funding organization had no role in the design or conduct of this research.

\section{Disclosure}

The authors report no conflicts of interest in this work.

\section{References}

1. Ramulu PY, Corcoran KJ, Corcoran SL, Robin AL. Utilization of various glaucoma surgeries and procedures in Medicare beneficiaries from 1995 to 2004. Ophthalmology. 2007;114(12):2265-2270.

2. Agbeja AM, Dutton GN. Conjunctival incisions for trabeculectomy and their relationship to the type of bleb formation - a preliminary study. Eye (Lond). 1987;1(6):738-743.

3. Brincker P, Kessing SV. Limbus-based versus fornix-based conjunctival flap in glaucoma filtering surgery. Acta Ophthalmol (Copenh). 1992;70(5): 641-644. 
4. Wells AP, Cordeiro MF, Bunce C, Khaw PT. Cystic bleb formation and related complications in limbus- versus fornix-based conjunctival flaps in pediatric and young adult trabeculectomy with mitomycin C. Ophthalmology. 2003;110(11):2192-2197.

5. Alwitry A, Patel V, King AW. Fornix vs limbal-based trabeculectomy with mitomycin C. Eye (Lond). 2005;19(6):631-636.

6. Fukuchi T, Ueda J, Yaoeda K, Suda K, Seki M, Abe H. Comparison of fornix- and limbus-based conjunctival flaps in mitomycin $\mathrm{C}$ trabeculectomy with laser suture lysis in Japanese glaucoma patients. Jpn J Ophthalmol. 2006;50(4):338-344.

7. Solus JF, Jampel HD, Tracey PA, et al. Comparison of limbus-based and fornix-based trabeculectomy: success, bleb-related complications, and bleb morphology. Ophthalmology. 2012;119(4):703-711.

8. Takihara Y, Inatani M, Fukushima M, Iwao K, Iwao M, Tanihara H. Trabeculectomy with mitomycin $\mathrm{C}$ for neovascular glaucoma: prognostic factors for surgical failure. Am J Ophthalmol. 2009;147(5): 912-918.

9. Takihara $\mathrm{Y}$, Inatani M, Seto T, et al. Trabeculectomy with mitomycin for open-angle glaucoma in phakic vs pseudophakic eyes after phacoemulsification. Arch Ophthalmol. 2011;129(2):152-157.

10. Iwao K, Inatani M, Seto $\mathrm{T}$, et al. Long-term outcomes and prognostic factors for trabeculectomy with mitomycin $\mathrm{C}$ in eyes with uveitic glaucoma: a retrospective cohort study. J Glaucoma. 2014;23(2): 88-94.
11. Awai-Kasaoka N, Inoue T, Takihara Y, et al. Impact of phacoemulsification on failure of trabeculectomy with mitomycin-C. J Cataract Refract Surg. 2012;38(3):419-424.

12. Inoue $\mathrm{T}$, Inatani $\mathrm{M}$, Takihara $\mathrm{Y}$, Awai-Kasaoka N, Ogata-Iwao M, Tanihara H. Prognostic risk factors for failure of trabeculectomy with mitomycin C after vitrectomy. Jpn J Ophthalmol. 2012;56(5): 464-469.

13. Broadway DC, Grierson I, Hitchings RA. Local effects of previous conjunctival incisional surgery and the subsequent outcome of filtration surgery. Am J Ophthalmol. 1998;125(6):805-818.

14. Law SK, Shih K, Tran DH, Coleman AL, Caprioli J. Long-term outcomes of repeat vs initial trabeculectomy in open-angle glaucoma. Am J Ophthalmol. 2009;148(5):685-695.

15. Beauchamp GR, Parks MM. Filtering surgery in children: barrier to success. Ophthalmology. 1979;86:170-180.

16. Cadera W, Pachtman MA, Cantor LB, Ellis FD, Helveston EM. Filtering surgery in childhood glaucoma. Ophthalmic Surg. 1984;15(4): 319-322.

17. Gressel MG, Heuer DK, Parrish RK 2nd. Trabeculectomy in young patients. Ophthalmology. 1984;91(10):1242-1246.

18. Yamamoto T, Kuwayama Y; Collaborative Bleb-related Infection Incidence and Treatment Study Group. Interim clinical outcomes in the collaborative bleb-related infection incidence and treatment study. Ophthalmology. 2011;118(3):453-458.
Clinical Ophthalmology

\section{Publish your work in this journal}

Clinical Ophthalmology is an international, peer-reviewed journal covering all subspecialties within ophthalmology. Key topics include: Optometry; Visual science; Pharmacology and drug therapy in eye diseases; Basic Sciences; Primary and Secondary eye care; Patient Safety and Quality of Care Improvements. This journal is indexed on

\section{Dovepress}

PubMed Central and CAS, and is the official journal of The Society of Clinical Ophthalmology (SCO). The manuscript management system is completely online and includes a very quick and fair peer-review system, which is all easy to use. Visit http://www.dovepress.com/ testimonials.php to read real quotes from published authors. 